\title{
Genomics: the power, potential and pitfalls of the new technologies and how they are transforming healthcare
}

\author{
Authors: Katherine S Josephs, ${ }^{A}$ Alison Berner, ${ }^{B}$ Angela George, ${ }^{C}$ Richard H Scott, ${ }^{D}$ Health Education England's \\ Genomic Education Programme, ${ }^{\mathrm{E}}$ Helen V Firth, ${ }^{\mathrm{F}}$ and Katrina Tatton-Brown ${ }^{\mathrm{G}}$
}

\begin{abstract}
Powerful new genomic technologies are transforming healthcare. The faster, cheaper generation of genomic data is driving the integration of genomics into all healthcare specialties. Within the next decade, healthcare professionals will be using genomic data to diagnose and manage their patients.

However, despite these exciting advances, few clinicians are aware of or prepared for this genomics-based future. Through five patient-focused scenarios with accompanying interviews, this article showcases new genomic technologies while highlighting the inherent challenges associated with complex genomic data.
\end{abstract}

KEYWORDS: Genomics, variant, next generation sequencing, personalised medicine, continuing medical education

\section{Genomics}

Genomic technologies are transforming healthcare with the facility to sequence more genes in shorter time periods and at ever reducing costs. Doctors and scientists today have an unparalleled ability to make gene discoveries, untangle molecular pathways

Authors: ${ }^{A}$ specialist registrar in clinical genetics, South West Thames Regional Genetic Services, London, UK and St George's, University of London, London, UK; ${ }^{\mathrm{B}}$ specialist registrar in medical oncology, Barts Cancer Institute, London, UK and clinical research fellow, Genomics England, London, UK; ' Consultant in oncogenetics, Royal Marsden NHS Foundation Trust, London, UK; ${ }^{\mathrm{D}}$ consultant in clinical genetics, Great Ormond Street Hospital for Children NHS Foundation Trust, London, UK and clinical lead for rare disease, 100,000 Genome Project, Genomics England, London, UK; ${ }^{\mathrm{E}} \mathrm{Health}$ Education England, London, UK; ${ }^{\mathrm{F}}$ consultant in clinical genetics, Cambridge University Hospitals NHS Foundation Trust, Cambridge, UK and Wellcome Sanger Institute, Cambridge, UK and Joint Committee on Genomics in Medicine, Royal College of Physicians, London, UK; ${ }^{G}$ consultant in clinical genetics, St George's University Hospitals NHS Foundation Trust, London, UK and professor in clinical genetics and genomic education, St George's, University of London, London, UK and Joint Committee on Genomics in Medicine, Royal College of Physicians, London, UK and find new targets for biomarkers and therapies. However, these genomic technologies are not without their associated challenges. If genomic data are to be integrated across healthcare, practitioners in all specialties will need to understand the potential pitfalls associated with the interpretation of genomic data to ensure they are used safely and for patient benefit.

Inspired by discussions at the Joint Committee on Genomics in Medicine (of the Royal College of Physicians, Royal College of Pathologists and the British Society of Genetic Medicine) and using five case studies with accompanying videos funded by Health Education England, we aim to illustrate the power and potential of genomics in clinical practice showcased by patients, families and their clinicians. In addition, through discussion with clinical scientists and mainstream colleagues, we will highlight some of the common difficulties that arise when interrogating genomic data.

\section{Case study 1: Genomic variants: innocent until proven guilty}

Patient 1 is a 12-year-old girl, referred to the genetics clinic with neonatal-onset tonic-clonic and absence seizures, microcephaly and profound developmental delay. A battery of investigations had been undertaken by the referring paediatrician, all of which were normal.

A gene panel, allowing the multiple parallel sequencing of 28 genes reported in association with early infantile epileptic encephalopathy, was performed to investigate an underlying genetic cause. Homozygous variants in the PNKP gene were identified: c.58C>T_p.(Pro20Ser). This particular variant had previously been published as pathogenic and in association with childhood epilepsy. ${ }^{1}$ However, detailed scrutiny of the literature identified phenotypic discrepancies between the 12-year-old patient and that of the other, reported, children.

The PNKP variant justified further investigation.

The genetic team evaluated the homozygous variant frequency in gnomAD, a freely accessible database curated by the Broad Institute and including genome and exome data from approximately 140,000 individuals, excluding those with paediatric disease (https://gnomad.broadinstitute.org). This variant is present homozygously in nine individuals included in the database. ${ }^{2}$ Only 17 individuals with PNKP-associated epilepsy have been reported to date; all have a severe childhood presentation inconsistent with inclusion in the gnomAD database. ${ }^{3}$ This variant is therefore too common in the general population to be classified 
as pathogenic. The variant has now been re-classified as benign and the family has been informed.

Given this re-classification of the PNKP variant, the original gene panel was re-analysed and a second variant was identified, this time in the SCN2A gene: c.2619C>G_p.(Ile873Met). This variant is not reported in gnomAD, has arisen de novo, is consistent with the child's phenotype and is therefore now assumed to be causative of this child's condition.

Dr Richard Scott, consultant in clinical genetics, clinical lead for rare disease, 100,000 Genome Project:

It's very easy when presented with results from a genomic test to find something rare and to think 'well if it's rare then perhaps it is the cause of this rare condition'. People are making really important decisions based on the information you give them and often there isn't enough evidence to be sure that a genetic variant is either the cause or not the cause of a condition.

Link to video: https://vimeo.com/336811697.

\section{Case study 2: The power of a genetic diagnosis}

Arvin's parents first became concerned when he was 7 months old and they noticed a change in his behaviour: they described that he stopped making eye contact and became less interactive. At the age of three, he was diagnosed with autistic spectrum disorder and a moderate intellectual disability. He was referred to clinical genetics and genetic investigations included a detailed analysis of his chromosomes (an array comparative genomic hybridisation $(\mathrm{CGH})$ ) and a gene panel which involved the parallel sequencing of 72 genes known to be associated with early onset epilepsy. He also underwent extensive mitochondrial and neurometabolic investigations. These tests did not identify an underlying cause for his condition.

At the age of five, Arvin was re-referred to genetics as his seizures were increasing in frequency and severity. At the time, his mother was 8 weeks pregnant and anxious about recurrence risks for her new baby. An urgent clinical exome, utilising new genomic technologies to sequence the coding regions (exons) of genes known to be associated with disease, was undertaken and a DHDDS gene variant was identified: c.110G>A_p.(Arg37His). The variant had arisen for the first time in Arvin (ie it was de novo), was absent from the gnomAD population database, is in a protein domain and predicted to be deleterious by multiple in silico tools. In addition, there is a single report in the medical literature describing two other children with the exact same variant and similar clinical features to Arvin. ${ }^{4}$ The variant was classified as pathogenic and the likely cause of Arvin's phenotype.

This result ended the family's 5-year search for a diagnosis. It enabled Arvin to access additional support at school and has potential implications for management: current data suggest that DHDDS-related seizures may respond better to sodium valproate than other anti-epileptics. ${ }^{4}$ However, of critical importance, prenatal diagnosis to investigate recurrence secondary to germline mosaicism (estimated at an empirical $<1 \%$ ) was now possible. The new baby was shown not to have inherited the DHDDS gene variant. Beskida, mother of Arvin, patient:

I was told the baby doesn't carry the gene [variant]. If I hadn't done the sequencing test, it would have been totally different. A big weight has been carried off my shoulders.

Link to video: https://vimeo.com/336803720.

\section{Case study 3: Interpreting results: beware the amplification of error}

Patient 3 was referred for predictive testing for a BRCA2 variant identified in her sister. She was 41 years old. Both Patient 3 and her sister were unaffected by cancer themselves, but testing in the sister had been undertaken in another country because several of her family members had died from early onset breast and ovarian cancer. Disease causing variants in BRCA2 cause an increased risk of both of these tumour types. On the basis of her results, patient 3's sister had elected to have risk reducing bilateral mastectomy and planned in the future to have a bilateral salpingo-oophorectomy.

Patient 3, keen to consider risk reducing surgery if she were found to carry the same BRCA2 variant as her sister, was referred to clinical genetics. However, scrutiny of the BRCA2 variant during the clinical genetics review confirmed the variant was currently classified as of uncertain significance and not clearly diseasecausing. Predictive testing was therefore not offered to her nor to her two daughters.

Nine months later, this particular variant was re-classified by Evidence-based Network for the Interpretation of Germline Mutant Alleles (ENIGMA; an international consortium aimed at determining the clinical significance of variants (https://enigmaconsortium.org)), as non-disease causing (benign).

Patient 3's sister had undergone unnecessary major surgery while patient 3 and her daughters have avoided uninformative genetic testing and unnecessary surgery. The underlying cause of the cancers in this family has not been established and it is still not clear whether the two sisters are at risk.

Dr Angela George, consultant in oncogenetics:

The difficulty when you receive a variant of unknown significance is how you use that information. You have to know the limitations of how you can interpret [genetic tests] and what should and shouldn't be done for these patients. We do have to be careful as clinicians that we are not making an error both for the patient in front of us ... but also amplifying any potential errors across their family members as well.

Link to video: https://vimeo.com/336813135.

\section{Case study 4: Access to gene-directed therapies}

Iain presented to his general practitioner at the age of 33 with a facial rash which he attributed to acne but which were angiofibromas, a hallmark of tuberous sclerosis complex (TSC). TSC is a complex, highly variable multi-system disorder characterised by hamartomas (benign tumours) that grow in the heart, brain, kidneys, skin and eyes and that can cause seizures, intellectual disability and autistic spectrum disorder. TSC is caused by mutations in the genes TSC1 and TSC2, which encode components of the mTOR signaling pathway, key for cellular growth and proliferation. ${ }^{5}$

Despite initial surveillance for kidney and brain lesions, Iain was lost to follow-up. Several years later, in his 50 s, he re-presented with a subconjunctival haemorrhage caused by uncontrolled hypertension. He was found to have renal angiomyolipomas (AMLs), one of the leading causes of morbidity and mortality in TSC patients. ${ }^{6}$

Everolimus has recently been licensed to treat adults with TSC-related AMLs. Everolimus is an mTOR pathway inhibitor and 
negatively regulates the pathway that $T S C 1 / 2$ gene mutations activate, thus reducing cell proliferation and the growth of AMLs. ${ }^{7}$ Given the size and number of AMLs that Iain had, he was eligible for recruitment to a national everolimus trial. He has recently commenced everolimus and hopes it will slow the growth of his AMLs, preventing complications, deterioration in his renal function and the need for surgical intervention.

Iain, patient:

The fact that this medication exists is fantastic. I think it is enormously powerful we have got to this point.

Link to video: https://vimeo.com/336804811.

\section{Case study 5: The realisation of personalised medicine}

Patient 5 is a 43-year-old man with no family history of cancer who presented with weight loss and jaundice. Abdominal imaging revealed a large liver mass and thickening of the bowel wall. A subsequent biopsy confirmed a moderately differentiated colorectal adenocarcinoma.

Tumour cells are characterised by thousands of gene mutations and chromosomal rearrangements acquired through the evolution from normal cell to tumour cell. The patterns of mutations within a tumour can represent specific mechanisms driving tumourigenesis. This pattern is known as a tumour's 'mutational signature'. Increasingly, genomic sequencing performed on the tumour tissue itself is being undertaken to reveal these signatures. ${ }^{8}$

Patient 5 's sequenced biopsy specimen had a mutational signature consistent with defective DNA mismatch repair (MMR): if cells cannot repair the DNA errors they routinely acquire, these newly acquired mutations (known as somatic mutations) may confer a proliferative advantage over neighbouring cells. Despite his lack of family history, the mutational signature identified in this case raised suspicion that the patient may have an inherited predisposition to colorectal cancer. Further genetic testing of a blood sample identified a germline pathogenic variant in MSH6, a gene associated with Lynch syndrome, which causes an increased risk of colorectal cancer.

The finding of an MMR-associated mutational signature has enabled patient 5 to access potentially lifesaving immunotherapy through clinical trials. ${ }^{9}$ In addition, his family is now able to access predictive genetic testing for Lynch syndrome and, if at risk, appropriate screening and preventative therapy.

Dr Alison Berner, specialist registrar in medical oncology and clinical research fellow:

There are many dimensions to how genomic medicine is really driving better cancer treatment and treatment that is more personal to the individual.

Link to video: https://vimeo.com/336816796.

\section{Discussion}

We have entered a genomic era. The new genomic technologies have provided us with a window to our DNA. Increasingly we are deciphering our genomic data to diagnose rare disease, determine management and better stratify disease risk for extended family members.

Case study 2 demonstrates the diagnostic power of these new technologies where a clinical exome ended a five year diagnostic odyssey and numerous, costly investigations. By making diagnoses that would be difficult or even impossible to make clinically, genomic technologies can open the door to reproductive choices, additional support and treatment options. The power of genomic technologies to determine management is additionally exemplified by case study 4 where knowledge of disease-causing genes and molecular pathways has enabled the discovery of new therapeutic targets and the option to re-purpose previously licensed drugs. In addition, the tumour mutational signatures discussed in case study 5 are an exciting application of genomic technologies enabling us to better define tumour types to stratify treatment and ultimately realise the vision of personalised cancer treatment.

Through these case studies, we have also sought to highlight the inherent challenges of genomic technologies. Our genomes are packed full of variation (differences in the DNA sequence) and the relationship between genetic variation and disease is not straightforward. ${ }^{10}$ On average we each have $4-5$ million variants, 10,000 of these will alter a protein and $40-100$ will have arisen for the very first time in us. Determining the significance of an individual variant relies on robust phenotyping, segregation patterns in families, the frequency of that variant in the healthy population, in silico tools and functional studies. Case studies 1 and 4 both highlight where variant misinterpretation could have led to clinical consequences. Despite previous reports the variant initially identified in patient 1 was not pathogenic and, had further analyses by the clinicians not been undertaken, there may have been future repercussions such as prenatal testing for an incorrect diagnosis. For patient 4 , had her clinicians not realised that the identified variant was of uncertain significance, this would have resulted in amplification of error through the family. Given the extent of normal variation and the consequence of variant misinterpretation, we strongly advocate an 'innocent until proven guilty' approach to variant interpretation and rigorous variant evaluation in the context of phenotype and contemporary evidence. In addition, a referral to a clinical genetics service may be considered to evaluate both the clinical and molecular components of a serious genetic diagnosis to ensure that the genetic diagnosis is robust, and fully explains the clinical features. Referral will also ensure that the patient (and their family) receives a full package of care relating to this lifelong diagnosis including up-to-date advice on prognosis, management, treatment, genetic risk to other family members, reproductive options, research opportunities and support group contact details.

Marie Curie wrote, 'Nothing in life is to be feared, it is only to be understood. ${ }^{11}$ Genome sequencing is not to be feared. It is an exciting, game-changing technology that is transforming healthcare. However, it must be understood if it is to be used safely. This article aims to raise awareness of some of the associated complexities of genome sequencing so that patients presenting to all healthcare specialties can continue to benefit from the unparalleled power of the genome.

\section{Acknowledgements}

We would like to thank the patients and their families who agreed to share their medical histories, in particular the two patients who gave personal interviews. We are grateful to Daniel Coley, producer at Tinker Taylor Production Company for the production and editing of each film and to Amelia McPherson, educational resources editor - Genomics Education Programme, Health Education England for both organising and helping with the interviews. Finally we thank the Joint Committee on Genomics in Medicine - a joint committee of the Royal College of Pathologists, the Royal College of Physicians and the British Society for Genetic Medicine - for their support with this article. 


\section{Supplementary material}

Additional supplementary material may be found in the online version of this article at http://www.clinmed.rcpjournal.org:

S1 - A glossary of terms.

\section{References}

1 Carvill GL, Heavin SB, Yendle SC et al. Targeted resequencing in epileptic encephalopathies identifies de novo mutations in CHD2 and SYNGAP1. Nat Genet 2013;45:825-30.

2 Lek M, Karczewski KJ, Minikel EV et al. Analysis of protein-coding genetic variation in 60,706 humans. Nature 2016;536:285-91.

3 Trump N, McTague A, Brittain $\mathrm{H}$ et al. Improving diagnosis and broadening the phenotypes in early-onset seizure and severe developmental delay disorders through gene panel analysis. J Med Genet 2016;53:310-7.

4 Hamdan FF, Myers CT, Cossette P et al. High rate of recurrent de novo mutations in developmental and epileptic encephalopathies. Am J Hum Genet 2017;101:664-85.

5 Randle SC. Tuberous sclerosis complex: a review. Pediatr Ann 2017;46:e166-71.
6 Brakemeier S, Vogt L, Adams L et al. Treatment effect of mTORinhibition on tissue composition of renal angiomyolipomas in tuberous sclerosis complex (TSC). PLoS One 2017;12:e0189132.

7 Bissler J], Kingswood JC, Radzikowska E et al. Everolimus for angiomyolipoma associated with tuberous sclerosis complex or sporadic lymphangioleiomyomatosis (EXIST-2): a multicentre, randomised, double-blind, placebo-controlled trial. Lancet 2013;381:817-24.

8 Helleday T, Eshtad S, Nik-Zainal S. Mechanisms underlying mutational signatures in human cancers. Nat Rev Genet 2014;15:585-98.

9 Kalyan A, Kircher S, Shah H, Mulcahy M, Benson A. Updates on immunotherapy for colorectal cancer. J Gastrointest Oncol 2018;9:160-9.

101000 Genomes Project Consortium, A Auton, Brooks LD et al. A global reference for human genetic variation. Nature 2015;526:68-74.

11 Benarde MA. Our precarious habitat, 2nd edn. New York: WW Norton \& Co, 1973:5.

Address for correspondence: Prof Katrina Tatton-Brown, South West Thames Regional Genetics Service, St George's University Hospitals NHS Foundation Trust, Cranmer Terrace, London SW17 ORE, UK.

Email: k.tattonbrown@nhs.net

\section{Consent and confidentiality in genomic medicine}

\section{Genetic or genomic tests are increasingly used in everyday medical practice. Every clinical field will encounter such tests to a greater or lesser extent.}

Published in 2019, this third edition by the Joint Committee on Genomics in Medicine provides updated guidance on the use of genetic and genomic information in the clinic. Health professionals from all areas of medicine need to know and understand how consent and confidentiality issues may arise, and to understand the potential ways in which the use of genomic tests may change the nature of the relationship between healthcare professionals and patients.

Download the guidance at:

www.rcplondon.ac.uk/consent-confidentiality-genomic-medicine

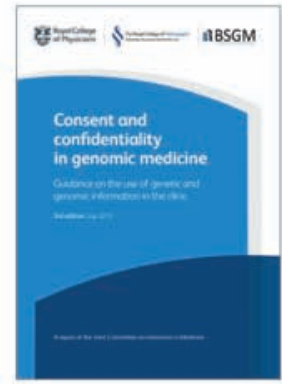

